\title{
SPATIAL FREQUENCY MASKING AND WEBER'S LAW
}

\author{
DAN J. SWIFT ${ }^{1.2}$ and Robert A. SMIth ${ }^{1}$ \\ ${ }^{1}$ Vision Research Laboratories of UNH. Pettee Brook Offices. Durham. NH 03824. U.S.A. and \\ ${ }^{2}$ Behavioral Sciences Department. University of Michigan-Dearborn. Dearborn. MI 48128. U.S.A.
}

(Receired 7 May 1980; in revised form 7 September 1982)

\begin{abstract}
The threshold masking effect of one simultaneously presented grating upon another was studied as a function of mask contrast and frequency. The masking function typically obeys Weber's Law with method-of-adjustment psychophysics. and typically does not with forced-choice. This apparent discrepancy was studied in some detail. We suggest that thresholds can be set with at least two different criteria. depending upon experimental conditions. When the mask is unfamiliar, it functions as noise and detection occurs at a constant signal/noise ratio, which yields Weber's Law. When the mask is highly familiar, its masking effect is less and obeys a power law. This power-law masking appears to represent an inherent non-linearity of the visual system.
\end{abstract}

\section{INTRODUCTION}

Considering the widespread use of masking techniques in the study of psychophysical thresholds, it is surprising that masking has only recently been applied to the study of spatial-frequency channels. In principle, the paradigm is extremely simple: the subject is presented with a superthreshold masking grating, and is asked to detect the presence of a superimposed test grating which is at or near threshold. Although this problem was studied as early as 1974 by Pantle (see Table 1), thorough parametric studies have not been published until recently (Legge and Foley, 1980). Possibly investigators have doubted their data because of a substantial discrepancy between the results reported by different groups. In many sensory modalities, experiments using masking or increment threshold tasks find that threshold elevation obeys the well-known Weber's Law: that is. increment threshold is proportional to background or mask intensity. Of the studies surveyed. about half find that Weber's Law holds, while the other hall find substantial and systematic deviations; typically threshold increases as some power of the masking contrast, with an exponent of about 0.65. Although the various studies differ in detail. they generally appear to be equivalent in principle. and it is by no means clear why this discrepancy occurs.

In a previous study (Swift and Smith. 1978), we suggested a simple model of the masking process. embodying three crucial assumptions which enabled us to deduce underlying channel properties from masking data. These assumptions were: (1) detection is done by a threshold mechanism which responds only to the output of a single. independent channel and is unafiected by other channels: (2) the mask's effect on the test-detecting channel is equivalent to the addition of an equal amount of noise: (3) detection occurs at a constant signal-to-noise ratio. with some unspecified internal noise mechanism determining absolute threshold. Assumption 3 implies that masking will obey Weber's Law. A number of earlier masking studies (e.g. Campbell and Kulikowski, 1968; Stromeyer and Julesz, 1972) appear to make similar assumptions, although these may be unstated. Since these assumptions, or some comparable set, are necessary if we wish to make quantitative inferences about channel tuning from masking data, we will devote the second part of this study to a critical examination of their validity.

\section{PART 1-PARAMETRIC MASKING DATA}

Legge and Foley (1980) have published a thorough, parametric set of masking data (test threshold versus mask contrast and frequency) using forced-choice psychophysics. Since psychophysical method appears to have a profound but unexplained effect on masking. we present similar parametric data using method-ofadjustment psychophysics.

\section{Methods}

The gratings used in this experiment were produced by conventional means on a Tektronix 535 oscilloscope with a P-2 phosphor. The screen had a mean luminance of $32 \mathrm{~cd} / \mathrm{m}^{2}$, and the area surrounding the screen was essentially dark. Viewing distance was $75 \mathrm{~cm}$ and the gratings were approximately $8 \mathrm{deg}$ wide by $6 \mathrm{deg}$ high. The spatial frequencies used were chosen not to bear exact integral relations to one another, so that there was no question of aligning the two superimposed gratings in any particular phase relationship. The mask and test gratings were present continually, and the subject adjusted the test grating to threshold by the method of adjustment. Each experimental session was devoted to a single pair of mask and test frequencies. It was immediately apparent to us that the psychophysical tasks involved in this experiment were difficult, and we found the results to be quite noisy. To deal with this we took an unusually large number of data (10-15 threshold 
Table 1. Survey of masking studies in which mask and test stimulus are reasonably similar

\begin{tabular}{|c|c|c|c|c|}
\hline Author & $\begin{array}{c}\text { Mask } \\
\text { temporal }\end{array}$ & $\begin{array}{c}\text { Test } \\
\text { temporal }\end{array}$ & $\begin{array}{c}\text { Spatial } \\
\text { frequency }\end{array}$ & Paradigm \\
\hline \multicolumn{5}{|c|}{ Studie's Finding Weher's Lau } \\
\hline $\begin{array}{l}\text { Smith and Swift } \\
(1978)\end{array}$ & Steady & Steady & $2-5 \mathrm{c} / \mathrm{deg}$ & Adjustment \\
\hline $\begin{array}{l}\text { Mostafivi and } \\
\text { Sakrison (1976) }\end{array}$ & Steady & Steady & 412 & $\begin{array}{l}\text { Adjustment: mask } \\
\text { was visual noise }\end{array}$ \\
\hline $\begin{array}{l}\text { Carlson and } \\
\text { Pica }(1979)\end{array}$ & - & $2 \mathrm{sec}$ & 12 & Forced choice \\
\hline $\begin{array}{l}\text { Campbell and } \\
\text { Kulikowski (1966) }\end{array}$ & Steady & Steady & 10 & Adjustment \\
\hline Legge (1979) & $200 \mathrm{msec}$ & $200 \mathrm{msec}$ & Many & $\begin{array}{l}\text { Forced choice. } \\
\text { dichoptic masking }\end{array}$ \\
\hline \multirow[t]{2}{*}{$\begin{array}{l}\text { Kulikowski and } \\
\text { Gorea }(1978)\end{array}$} & Steady & $\begin{array}{l}0.5 \mathrm{~Hz} \\
\text { on-off }\end{array}$ & $>7.5$ & Adjustment \\
\hline & $\begin{array}{l}8 \mathrm{~Hz} \\
\text { Reversal }\end{array}$ & $\begin{array}{l}8 \mathrm{~Hz} \\
\text { Reversal } \\
+0.5 \mathrm{~Hz} \\
\text { on-off }\end{array}$ & 0.6 & Adjustment \\
\hline Bodis-Wollner & Steady & $8 \mathrm{~Hz}$ on off & 12 & Yes No \\
\hline $\begin{array}{l}\text { Pollen and Roehrig } \\
(1970)\end{array}$ & Steady & Steady & $0.4-12$ & $\begin{array}{l}\text { Yes-no, mask } \\
\text { was visual noise }\end{array}$ \\
\hline $\begin{array}{l}\text { Stromeyer and } \\
\text { Julesz }(1972)\end{array}$ & Steady & Steady & Many & $\begin{array}{l}\text { Adjustment. mask } \\
\text { was visual noise }\end{array}$ \\
\hline \multicolumn{5}{|c|}{ Studies Not Finding Weber's Law } \\
\hline Legge (1979) & $200 \mathrm{msec}$ & $200 \mathrm{msec}$ & Many & $\begin{array}{l}\text { Forced choice. } \\
\text { monocular }\end{array}$ \\
\hline Pantle (1974) & Steady & $1.7 \mathrm{sec}$ & $2-10$ & Forced choice \\
\hline $\begin{array}{l}\text { Legge and } \\
\text { Foley (1980) }\end{array}$ & $200 \mathrm{msec}$ & $200 \mathrm{msec}$ & 2 & Forced choice \\
\hline $\begin{array}{l}\text { Nachmias and } \\
\text { Sansbury (1974) }\end{array}$ & $250 \mathrm{msec}$ & $250 \mathrm{msec}$ & 3 & Forced choice \\
\hline $\begin{array}{l}\text { Kulikowski and } \\
\text { Gorea (1978) }\end{array}$ & Steady & $0.5 \mathrm{~Hz}$ & $<7.5$ & Adjustment \\
\hline $\begin{array}{l}\text { Bodis-Wolliner } \\
\text { et al. (1973) }\end{array}$ & Steady & $8 \mathrm{~Hz}$ on-off & 1.5 & Yes-no \\
\hline $\begin{array}{l}\text { Tolhurst and } \\
\text { Barfield (1978) }\end{array}$ & $1 \mathrm{sec}$ & $1 \mathrm{sec}$ & 4.25 & Forced-choice \\
\hline
\end{tabular}

settings for each data point). The threshold for the test stimulus was measured for (typically) seven values of mask contrast, beginning with the lowest value and progressing through to the highest. At each mask contrast, five settings were made by the method of adjustment. Each pair of frequencies was run on at least two separate sessions and the results averaged. The subject took frequent breaks between thresholds, to avoid a build-up of spatial-frequency adaptation.

\section{Results}

A typical set of data is shown in Fig. 1a; this figure plots the threshold for the test grating as a function of the contrast of the mask. There are three significant portions of this masking threshold function. For very low mask contrasts, the threshold of the test stimulus is unaffected and is the same as the unmasked threshold. When the mask is, itself, near threshold there is actual facilitation or lowering of the test threshold. This does not occur under all conditions, as we shall see presently. When the mask grating is well above its threshold, test threshold rises rapidly, and under many conditions, test threshold is propor- tional to mask contrast (i.e. Weber's Law). Figure lb shows typical masking data when the mask and test frequencies are separated by about an octave. Certain differences are immediately apparent; in particular. the facilitation effect has disappeared, so that the curve has lost its characteristic dipper shape. At higher mask contrasts there is less threshold elevation, and threshold elevation does not begin until the mask grating is well above its own threshold. The slope of this portion of the curve has begun to decrease and is now clearly less than 1 .

Figures 2 and 3 show complete families of threshold elevation functions for a variety of mask frequencies. In general we see that Weber's Law applies when the mask and test frequencies are within one octave of each other, and that the facilitation effect is observed over a somewhat narrower range with a $5 \mathrm{c} / \mathrm{deg}$ test grating, while it is largely absent with a $3 \mathrm{c} / \mathrm{deg}$ test grating.

Several differences can be seen between our results and those of Legge and Foley (1980). The absence of subthreshold facilitation in our $3 \mathrm{c} / \mathrm{deg}$ condition is puzzling, and we have no explanation. Possibly this 


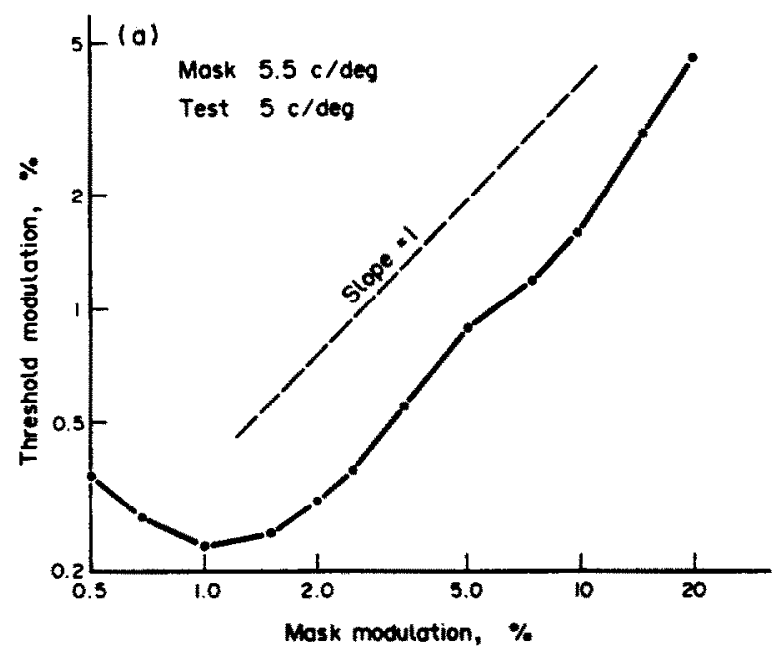

Fig. 1a. A typical masking function; this shows the effect of mask contrast on the threshold for the test grating, when the frequencies of the two gratings are similar.

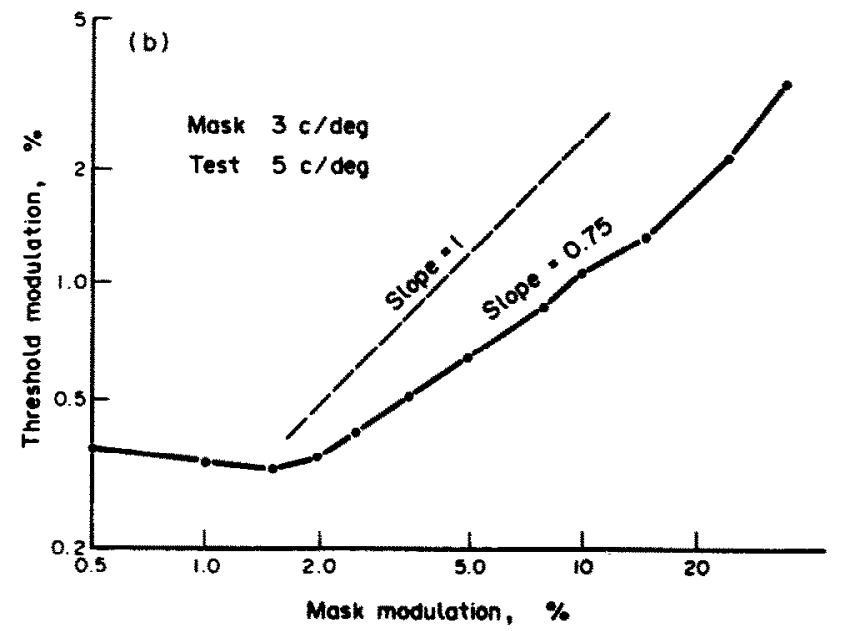

Fig. 1b. Another masking function: similar to Fig. 1a, but for mask and test frequencies which differ by about a factor of two.

effect is criterion dependent; these are very difficult discriminations and criterion shifts have been a continual problem. Legge and Foley did not find a decrease in the slope of the masking function with increased separation of mask and test frequency; however their slopes were all somewhat less than ours, and their range of mask frequencies was not as great. Finally they found that masking obeyed a power law with an exponent of 0.6 under all conditions, where most of our data obey Weber's Law. We find this to be the most interesting of the discrepancies and one which pervades the literature, so we made it the focus of further study.

\section{PART II-INVESTIGATION OF WEBER'S LAW AND THE MECHANISM OF DETECTION}

In the introduction, we noted three assumptions which have commonly been made in the study of spa- tial masking. The first of these is the assumption that detection occurs when the excitation in the channel tuned to the test frequency exceeds some threshold. In the following section we will show that this singlechannel threshold criterion is inadequate and that discrimination must be done by considering the relative excitation in many channels. In the final section of this paper we will consider-in the context of the Weber's-Law/power-law controversy-our two remaining assumptions, which postulate that the mask may be regarded as noise in a detector limited by the signal-to-noise ratio.

\section{Methods}

A much improved apparatus was used for all further experiments. The following is a brief description; a complete description of both the apparatus and the psychophysical techniques is in preparation. Gratings were displayed on a Hewlett Packard Model 


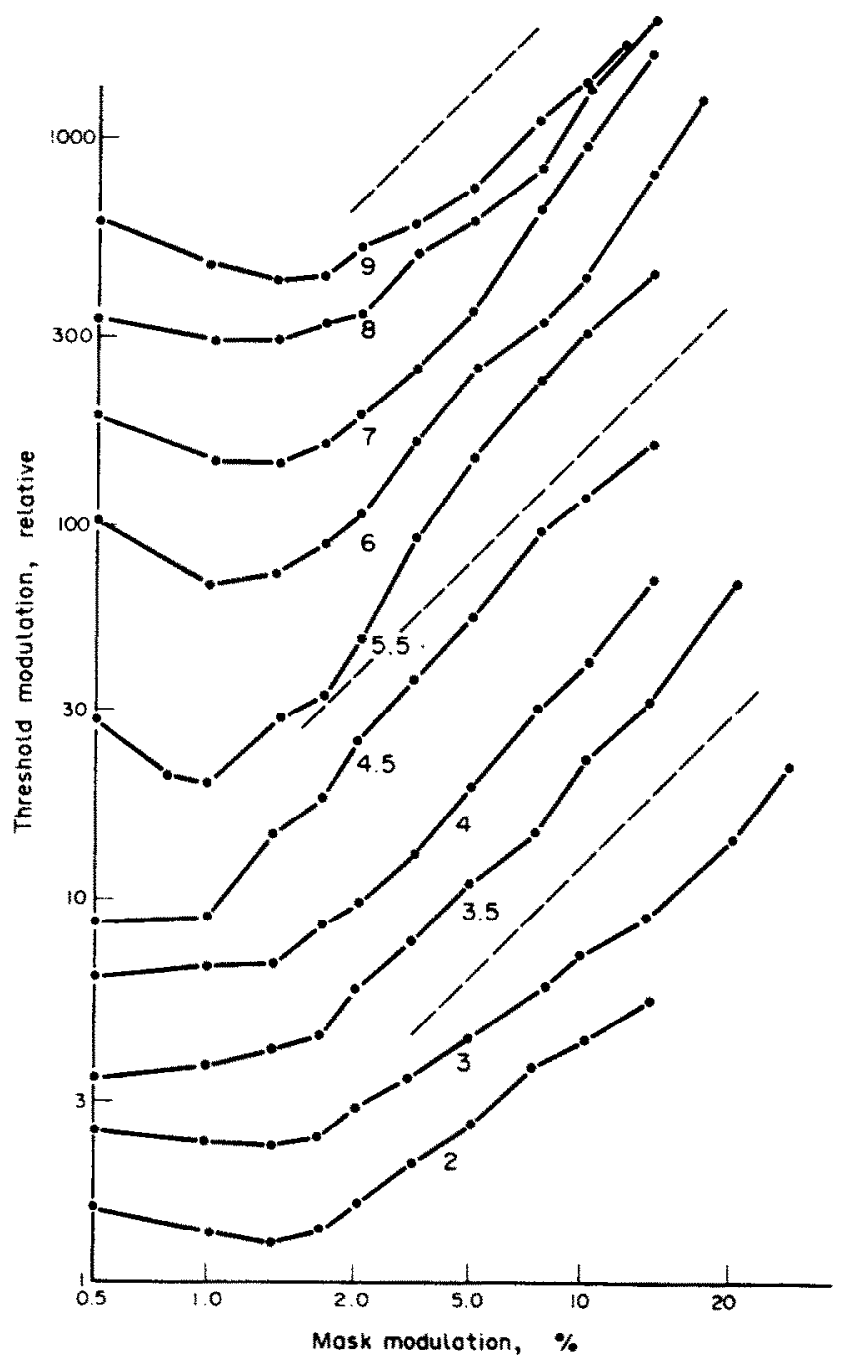

Fig. 2. A complete set of masking functions for a test frequency of $5 \mathrm{c} / \mathrm{deg}$. The leftmost point of each curve is the same $\left(0.36^{\circ}{ }_{0}\right)$ : the curves have been translated upward for clarity. Each curve is labeled with its mask frequency.

$1332 \mathrm{~A}$ display, with a luminance of $65 \mathrm{~cd} / \mathrm{m}^{2}$ and a P-4 phosphor. This was driven by a Xitan Z-80 micro-computer with an interface of our own design, operating at 80 frames per second. Superimposed mask and test gratings were produced by alternating mask and test on successive frames. No flicker was apparent using this procedure. The display was viewed from a distance of $75 \mathrm{~cm}$ and was approximately 6 deg high by 8 deg wide. Stimuli were viewed binocularly and subjects used free fixation.

The stimuli were calculated by the micro-computer, which also stored the observer's responses. Most of the data was collected with a forced-choice technique of our own design. In this technique. the intensity of the test stimulus decreases by one step whenever the subject responds correctly, and increases by 5 stcps whenever the response is incorrect. The step size is approximately one standard deviation of the psychometric function underlying the discrimination (in this case, $\left.0.2^{\circ}{ }_{0}\right)$. The forced-choice staircase continues until seven errors have been recorded. The contrasts at which errors occurred are then ordered and averaged with the following weightings (lowest: $0,0.05$. $0.1,0.2,0.3,0.25,0.1$ : highest). These weights were determined in preliminary studies to yield a threshold at the $75_{\%}^{\circ}$ point of the psychometric function, and an error of about one-half standard deviation of the psychometric function. The procedure is considerably faster than other forced-choice procedures we have used.

A typical successive forced-choice trial had the following sequence. The display began with a $3-\mathrm{sec} \mathrm{ex}$ posure to a background (either a grating or a blank field). This was followed by a 400 msec presentation of the mask followed by $1.2 \mathrm{sec}$ of background. After this a second mask was exposed for $400 \mathrm{msec}$, followed by a final background of $800 \mathrm{msec}$. The test grating was added to one of the masks, chosen randomly. There 


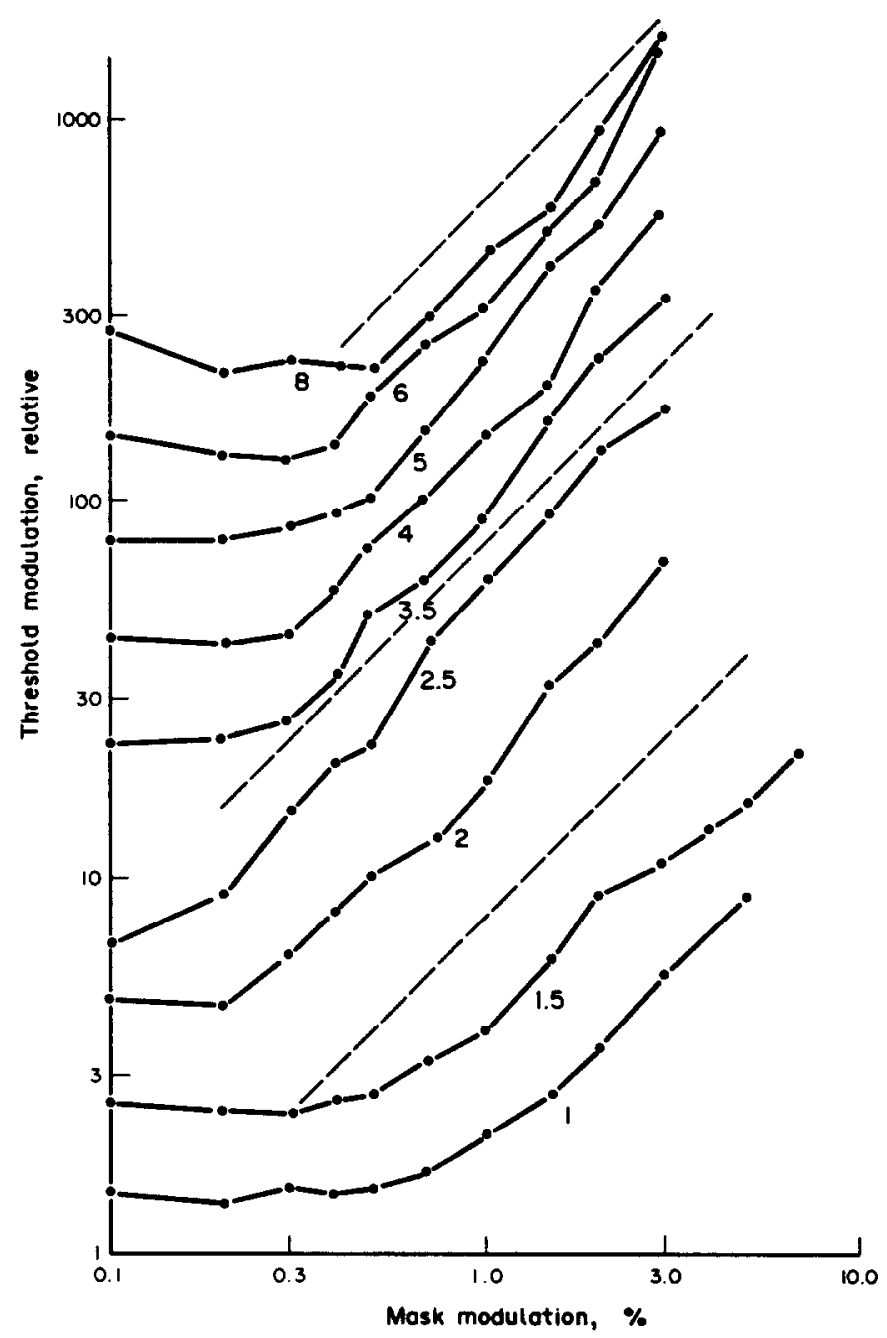

Fig. 3. Similar to Fig. 2 for a test frequency of $3 \mathrm{c} / \mathrm{deg}$. The leftmost point of each curve is the same $\left(0.25^{\circ}\right)$ : the curves have been translated upward for clarity.

was a delay of one-half second between tests, during which the screen was blank and the observer indicated which interval contained the test-and-mask (vs mask-alone) stimulus.

On a typical simultaneous forced-choice trial, the subject was presented with side-by-side test-and-mask and mask-alone stimuli. Each was approx $3 \mathrm{deg}$ wide and they were separated by one-half deg. The stimuli were present indefinitely, until the subject indicated his choice (typically less than $2 \mathrm{sec}$ ), whereupon they vanished for one-half sec while another stimulus was being computed.

\section{The relative detection criterion}

The earliest and best known of the multiple-channel models (Campbell and Robson, 1968) postulates independent channels, each with a separate thresholdtype detector. Legge and Foley (1980) have used such single-channel threshold detectors in their highly successful masking model. For many purposes, such a detector is adequate, but for detecting complex threshold stimuli it has been found to be seriously over-simplified (Graham, 1977). In this section we present evidence to show that detection in the presence of a mask is based on the relative outputs of a number of channels and cannot be explained by a single-channel threshold model.

\section{Contrast-compensation experiment}

One approach to this problem is provided by what we call a contrast-compensation experiment. In this experiment we decrease the contrast of the mask component of the mask-plus-test stimulus (but not the mask-alone stimulus) by an amount proportional to the contrast of the test stimulus. This decrement should reduce the stimulation in the test channel, thereby partially or totally cancelling the increase in stimulation due to the presence of the test stimulus. The net result, under the single-channel threshold model, should be to reduce the effect of the test stimulus and to make discrimination more difficult. A quantitative prediction of this effect is possible only in 


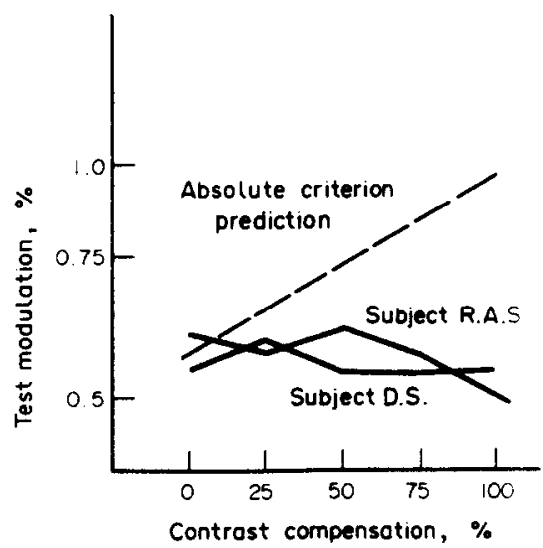

Fig. 4. The effect of contrast compensation (see text) on test threshold. Mask frequency $3 \mathrm{c} / \mathrm{deg}$. contrast $12.5^{\circ}$. Test frequency $4 \mathrm{c} / \mathrm{deg}$. The dotted line shows the prediction of Legge's single-channel-threshold model.

the context of a detailed model of the masking process; we have used Legge and Foley's (1980) model for this purpose.

The experiment was run using our standard simultaneous forced-choice procedure (see "Methods") with the following modification. The contrast of the maskalone stimulus was presented at its specified value of $12.5 \%$. The contrast of the mask component of the mask-plus-test stimulus was decremented by an amount proportional to the contrast of the test stimulus. The constant of proportionality determined the amount of compensation (e.g. $50 \%$ compensation meant that the mask contrast was reduced by an amount equal to half of the test contrast). Thresholds were measured for a $4 \mathrm{c} / \mathrm{deg}$ test against a $3 \mathrm{c} / \mathrm{deg}$ mask, as a function of the amount of compensation. Using Legge and Foley's model, we calculated thresholds for both the channel most sensitive to the test and the channel most sensitive to the mask. In fact, the test channel was always significantly more sensitive, so the presented results reflect that channel alone.

The results of the experiment and the simulation are shown in Fig. 4. As expected, the single-channel threshold model predicts that discriminability will decrease dramatically as compensation increases. In contrast to the prediction. however, the data for two subjects are essentially flat, convincingly arguing for some other criterion. We have insufficient information to specify the exact nature of this criterion. but it would appear that it must necessarily involve the relative outputs of at least two channels. We have successfully modeled the results of this experiment using a criterion requiring that the output of the test channel must exceed the mean output of all channels by some threshold amount. This is only a suggestion, however; no doubt other equally satisfactory criteria could be devised.

\section{Interstimulus-interval experiment}

There is another approach to the single-channel- threshold hypothesis, which yields further insight. Two related paradigms in the masking literature are sometimes confused, probably inappropriately. We shall call these true masking and contrast discrimination (Legge. 1981). In true masking. the mask and test frequencies are different: in contrast discrimination they are the same. While it is clear that contrast discrimination can logically be regarded as a special case of masking. it is quite possible that it is treated differently by the visual system. In particular. it is clear that detection in the contrast discrimination situation must be done by the criterion of incremental stimulation to the test channel, since mask and test excite the exact same narrow band of channels. The stimulation of a variety of channels in the true masking situation, however. makes it possible that discrimination is done by a more complex pattern analysis. We hypothesize that this is the case and furthermore hypothesize that the true masking discrimination is of a qualitative nature: either a particular pattern configuration is detected by the subject or it is not. This hypothesis may be tested. The subject is asked to do contrast discrimination and true masking judgments in a successive forced choice paradigm. As the interstimulus interval (ISI) is made progressively longer, it is clear that a quantitative discrimination based on remembered relative stimulus intensity will be degraded to a greater extent than will a more qualitative discrimination. The results of such an experiment are shown in Fig. 5, where the prediction is verified. Increasing ISI from four to ten seconds sharply raises the threshold for contrast discrimination while producing only a minor effect with true masking. This reinforces our assertion that a criterion based on the stimulation in a single channel cannot be responsible for all masking results. It also makes the point that the contrast discrimination situation should probably be considered separately from true masking and that results obtained with the two paradigms may differ in fundamental ways. With a few explictly noted exceptions, we have previously avoided the contrast discrimination paradigm. and shall continue to do so for the remainder of this study.

\section{PART III-WEBER'S LAW OR POWEK LAW?}

In Table 1 we present experimental parameters from a number of studies of spatial-frequency masking. The results in this table are all for the case in which mask and test frequency are either equal or very close (a requirement which has caused the omission of several well-known studies). As one scans Table 1, certain generalizations become clear. It is apparent that there is a loose association between Weber's law and the use of method-of-adjustment psychophysics. It is likely that the more sustained stimulus presentation characteristic of this method would primarily stimulate the sustained channels of the visual system. (For a discussion of the sustained 


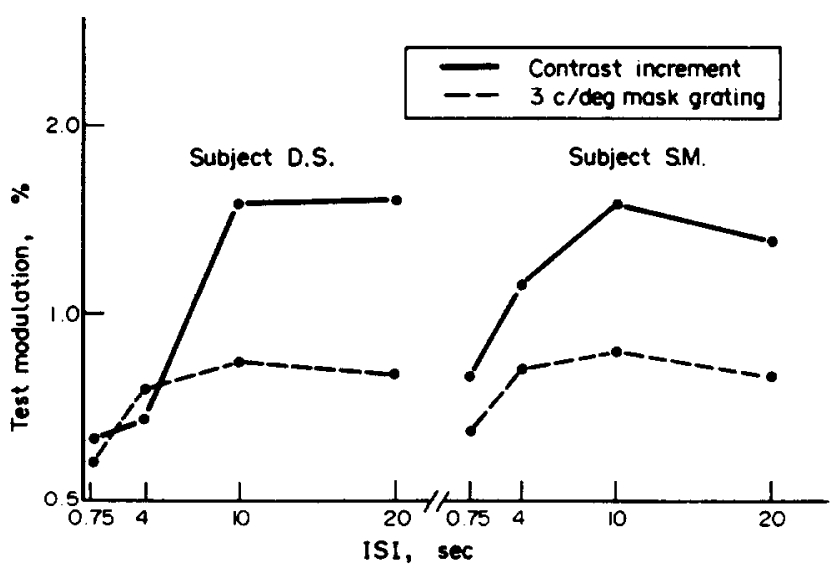

Fig. 5. The differential effect of ISI on contrast discrimination $(4 \mathrm{c} / \mathrm{deg})$ and true masking $(3 \mathrm{c} / \mathrm{deg}$ mask. $4 \mathrm{c} / \mathrm{deg}$ test). The standard error is, on average, 0.32 of the threshold.

and transient channels hypothesis, see Legge, 1978.) This suggests a first, tentative hypothesis.

\section{Hypothesis I}

Weber's Law is a property of the sustained visual channels, and does not apply when the transient channels are substantially stimulated.

This hypothesis is attractive because it agrees with studies of temporal contrast detection; Kelly (1961) found Weber's Law to hold only at very low temporal frequencies (sustained system). However, Kulikowski and Gorea (1978) explicitly tested this hypothesis, using a stimulus designed to maximally stimulate the transient system, and convincingly obtain Weber's Law. Their adapt stimulus was a $0.6 \mathrm{c} / \mathrm{deg}$ grating, flickered in counterphase at $8 \mathrm{~Hz}$. The test stimulus was identical to the mask (effectively a contrast increment) and was turned on and off at a rate of $0.5 \mathrm{~Hz}$.

\section{Hypothesis II}

Weber's Law occurs if those visual channels used to detect the test stimulus are all stimulated by the mask stimulus.

It is necessary to consider more than just spatial frequency in applying this hypothesis. Kulikowski and Gorea suggest that we must also consider the distinction between sustained and transient channels. This hypothesis may be a necessary (but not sufficient) condition; we know of no convincing case in which Weber's Law is found to hold when the test stimulus would be expected to excite channels which are not masked. However, the data of Legge and Foley (1980) and some of our own data (below) show that Weber's Law does not always apply even when mask and test stimuli are apparently identical, and so must necessarily excite the same channels.

\section{Hypothesis IIJ}

Weber's Law occurs only when the mask stimulus produces essentially complete spatial-frequency adaptation of the channels which detect the test stimulus.
This hypothesis was proposed by Bodis-Wollner et al. (1973) and tested extensively by Kulikowski and Gorea (1978). It is consistent with a great many of the data.

\section{Hypothesis IV}

The experiments in Table 1-while seemingly equivalent - reflect at least two fundamentally different detection strategies. Factors which cause the observer to use different strategies include (1) whether the mask frequency is or is not equal to the test frequency and (2) the psychophysical method used.

Experimental test of adaptation as a necessary condition for producing Weber's Law

Consider Hypothesis III, that Weber's Law holds if and only if the visual system is in a state of complete spatial-frequency adaptation. We further tested this hypothesis by replicating and extending Kulikowski and Gorea's counter-phase masking experiment. Such a replication, using successive forced-choice technique, is shown in Fig. 6, where we verify their observation of Weber's Law. We now attempted a varjation on the paradigm; the counter-phase mask stimulus was presented only during test periods. Since the mask stimulus will now be present only about $10 \%$ of the time, it will produce little adaptation, and should not produce Weber's Law if Hypothesis III is correct. In fact, the results are quite indistinguishable from the previous case, clearly disproving BodisWollner et al.'s hypothesis. As a control, we measured the amount of spatial frequency adaptation produced by the two masking stimuli. Experimentally, the distinction between masking and adaptation is that adaptation is measured when the mask stimulus is not present. In practice therefore, it is not possible to maintain the mask stimulus at a $90 \%$ duty cycle, as was the case in the first masking experiment. However, by shortening the intervals between and after the test periods, we were able to achieve a $65 \%$ duty cycle. while maintaining the original 6-sec trial length. 


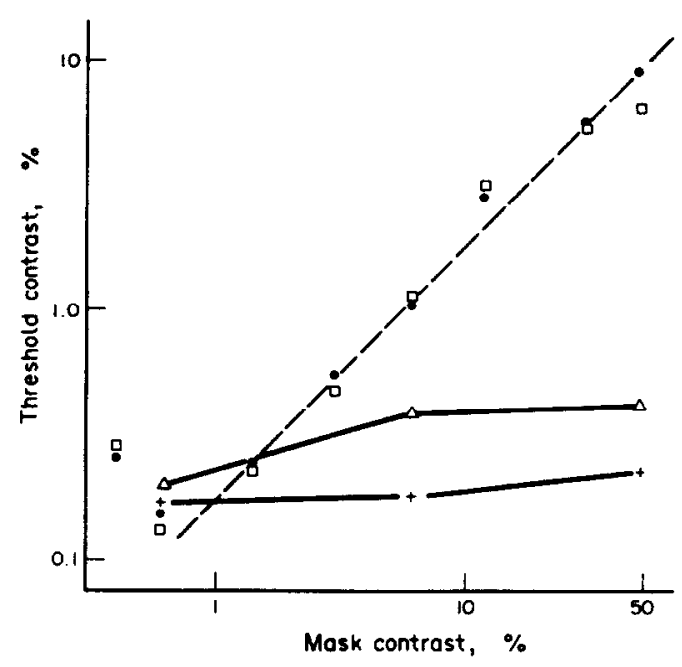

Fig. 6. Forced choice masking by Kulikowski and Gorea's counterphase stimulus $(0.6 \mathrm{c} / \mathrm{deg}, 8 \mathrm{~Hz}$ counterphase flicker) for sustained $(\square)$ and transient ( $\bullet$ ) mask conditions. Adaptation produced by the two mask stimuli for sustained $(\Delta)$ and transient $(+)$ mask conditions.

Measuring adaptation to the transient mask stimulus was not a problem; the mask was presented for $800 \mathrm{msec}$, as in the original experiment, ending $1000 \mathrm{msec}$ before the first test period. It will be seen (Fig. 6) that the transient mask produces little adaptation while the longer mask produces adaptation of about a factor of 2. A two-fold increase in threshold is typical of spatial adaptation, when measured with forced-choice psychophysics (Swift et al., 1979a).

Thus we see that the presence of spatial adaptation is not a necessary condition for Weber's Law, at least in this experiment. Adaptation is not a sufficient condition for Weber's Law, either. Using simultaneous forced-choice psychophysics, we took many data in which the mask and test stimuli were present with about a $90 \%$ duty cycle, so that complete adaptation would be expected to occur. Although the details will be discussed later, the general conclusion is quite clear: using a $4 \mathrm{c} / \mathrm{deg}$ test, we could find no conditions whatsoever in which an harmonically pure mask produced Weber's Law. Legge (1981) reported similar findings. These results demonstrate two important points: (1) Adaptation is neither a necessary nor a sufficient condition for Weber's Law; (2) Comparing these results with results from Part I suggests that psychophysical method alone may determine whether Weber's Law is observed. Thus, consideration of the first three hypotheses, though instructive, shows all of them to be inadequate. We must now consider the final hypothesis.

\section{Learning experiment}

The key to understanding the non-occurrence of Weber's Law lies in the extensive learning effects observed whenever a subject is faced with a new masking task (cf. Nachmias and Rogowitz, 1982). Fioren- tini and Berardi (1981) studied grating discrimination and reported that thresholds decrease over the course of 200 trials. We find that not only do thresholds decrease with practice on a new paradigm. but the slope of the masking function typically decreases from about 1 to about 0.65 . This phenomenon-long unrecognized by us-caused considerable confusion in our search for conditions which produced Weber's Law. As we tried each new paradigm. it at first appeared that we had finally succeeded. only to have our results slowly degenerate to the familiar 0.65 power law. We began systematically studying the process of learning a new masking task. However. changing the experimental parameters (e.g. presentation order) to produce a new task-to-be-learned is a poorly-controlled process at best. We found that we could create different tasks at will by using different random-noise masks. Our noise patterns were the sum of eight sinusoids of equal amplitude spaced at equal log intervals over the range of $2-8 \mathrm{c} / \mathrm{deg}$; phases of the eight components varied at random between different patterns. Since this learning is a one-time phenomenon. not easily replicated and averaged, we present data in detail for subject RS, learning to detect the $4 \mathrm{c} / \mathrm{deg}$ test in the presence of four different noise patterns (Fig. 7). The session starts with a careful measurement of the subject's masking function, each point being derived from a single simultaneous $2 A F C$ session (see "Methods"). Five points were obtained initially, and they clearly display a slope of 1 . From this, two convenient points were selected for repeated settings. As RS continued to set his threshold for these two points (always with the same mask pattern). settings decreased systematically as did the slope of the line joining the two points. Eventually this slope reached a value of 0.65 . At this point a new random noise mask was selected, and the slope again rose to 1.0. falling off as before with repeated settings. RS appears to have developed a learning set: he requires fewer and fewer trials to reach asymptote. and eventually is able to do so on the first trial. We suspect that learning still occurs, but it is too fast to be seen. Figure 8 shows similar data for two additional observers. These observers learned more slowly than RS, so each point represents the mean of 4 trials. The overall decrease in threshold is much less pronounced here. probably because it occurs in the first few trials. Despite these differences, however, the decrease in slope is still observed. Thus we conclude that there is a genuine learning phenomenon involved in masking and that as learning proceeds masking tends to change from Weber's Law to power law. The substantial individual differences observed suggest a detailed study of this learning process. In particular, we suspect the existence of measurable correlates of various subjective phenomena, such as choice of criterion.

It appears from the results of the previous experiment that familiarity with the mask stimulus may be crucial in determining the occurrence of Weber's Law or power law behavior; with increased familiarity de- 


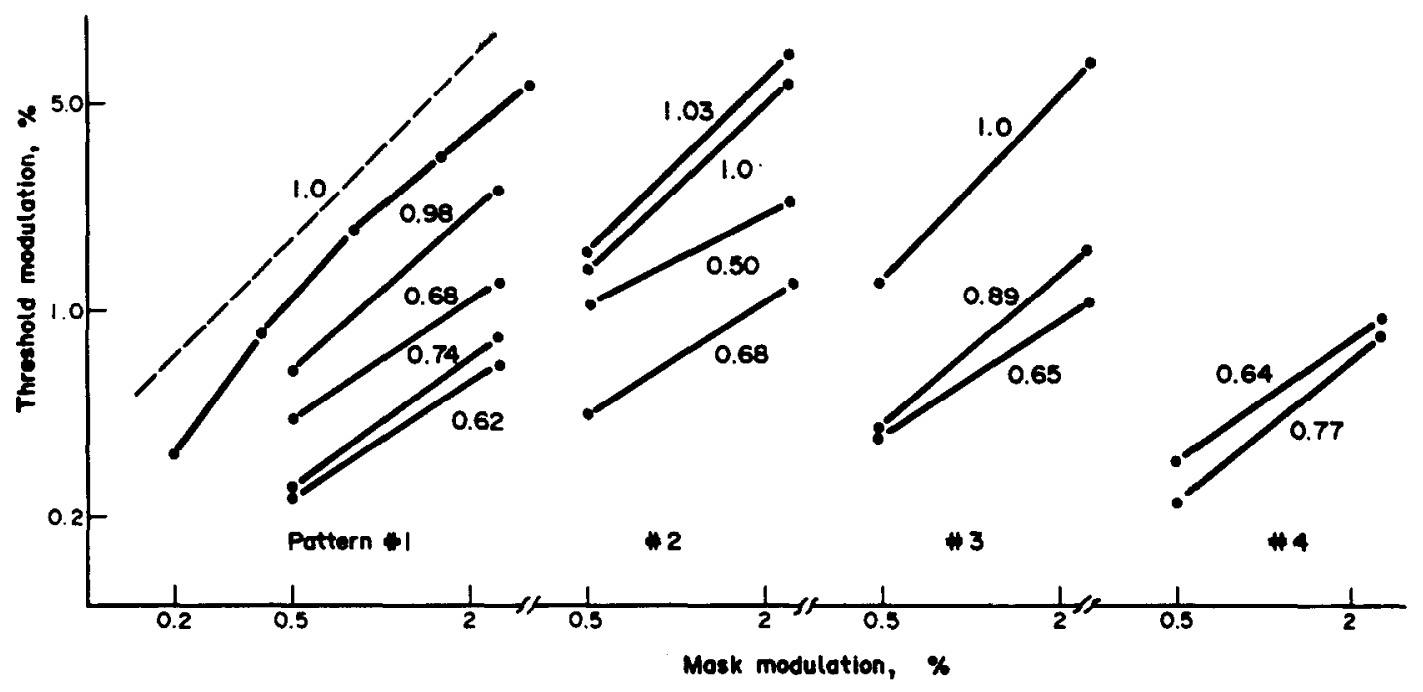

Fig. 7. Learning to detect a $4 \mathrm{c} / \mathrm{deg}$ test in the presence of 4 different random masks. Subject RS. In each column, the highest curve was run first (curves are not displaced).

tection seems to change from one to the other. If the subject could be prevented from becoming familiar with the mask stimulus, then Weber's Law behavior should persist indefinitely. We tested this by repeating the experiments of the preceding paragraph with one change in procedure; on each and every trial a new masking pattern was used, so that the subject saw each mask pattern only once, for the two stimuli of a particular trial. The results of this experiment are shown in Fig. 9, where we have plotted the slope of the masking function versus the number of trials. It will be seen that for the changing-mask condition the slope does not differ significantly from 1.0 over a period of many trials, but if the same mask pattern is used repeatedly (data replotted from Figs 7 and 8) then the slope quickly falls off to a value of about 0.65 . These results seem to confirm our hypothesis that-in this paradigm at least-it is familiarity with the mask stimulus which determines the functional form of the masking.

We believe that this result can be interpreted in terms of the information content of the mask. If a mask is familiar to the subject then it effectively contains no information. Such a mask can be discounted if the subject looks for small deviations from the expected appearance of the mask. It was subjectively quite clear to our subjects that this was happening. Over the course of many trials, the subject would learn to use a particular local criterion for making any given discrimination. This criterion would vary

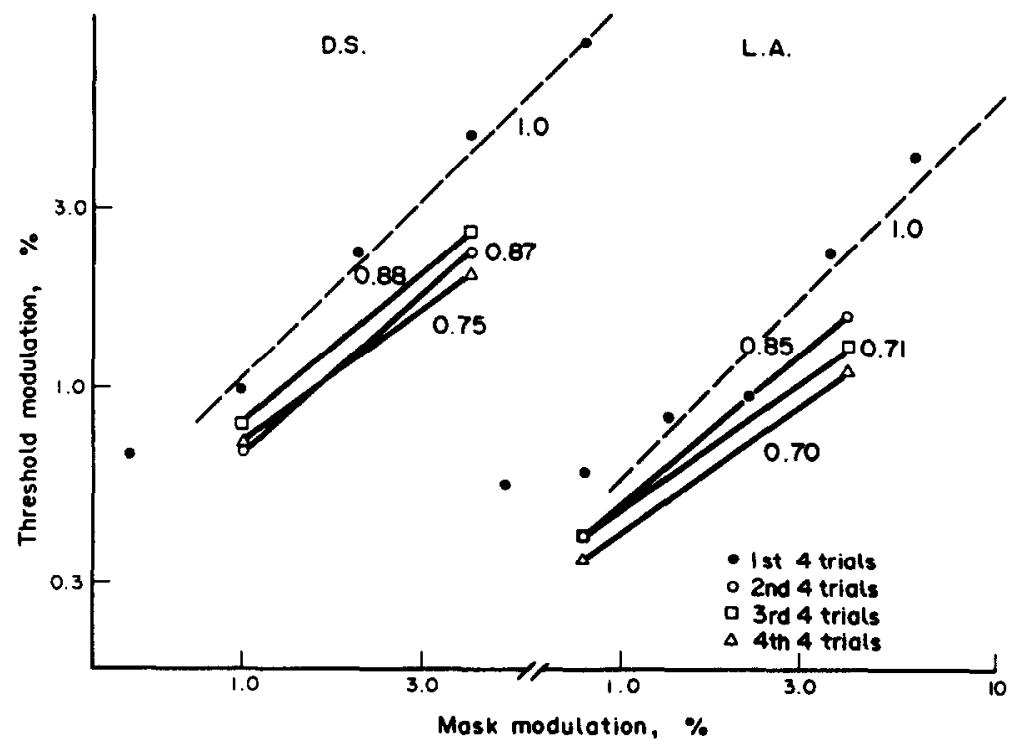

Fig. 8. Similar to Fig. 7 for subjects DS and LA. Here each point is the average of 4 trials. 


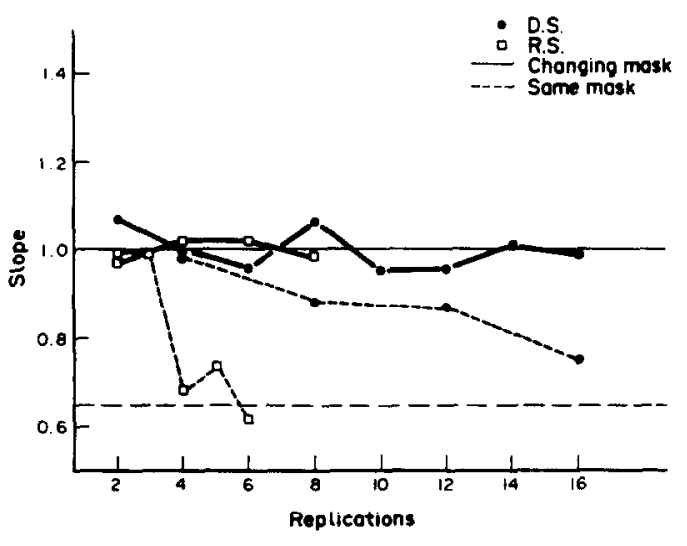

Fig. 9. The slope of the masking function (e.g. Figs 7 and 8) versus number of trials practice. Solid lines are for the case where the mask was changed on every trial. dotted lines are for when the mask was not changed.

with different masks, and had to be discovered by the subject for each new mask. A typical criterion might have been to compare the relative brightnesses of two prominent bars in the masking noise pattern, and seeing if this differed from what was expected for the mask alone. (For a similar result, see Nachmias and Weber, 1975). The subjective process of learning the appropriate criterion was paralleled by a decrease in threshold towards a final asymptote.

\section{DISCUSSION}

What are we to conciude about the mechanism of masking and Weber's Law from these results? We suggest that the results indicate the existence of several distinct detection mechanisms. In the case of contrast-increment detection, detection must be based upon absolute changes in stimulation to those few channels which respond to the test frequency. since no other frequency is present. However, where the mask pattern is different from the test pattern, detection may be done by comparing relative excitations in different channels (in a way as yet unknown) and not by considering excitation only in a single channel. This was demonstrated conclusively by the results of the contrast-compensation and ISI experiments.

In the case of masking by random noise. we were able to distinguish two different threshold criteria. These appear to be variants of the relative threshold criterion mentioned in the previous paragraph. We believe it is the unintentional admixture of singlechannel and multiple-channel strategies throughout the literature which is responsible for the sometime observance or nonobservance of Weber's Law. This is tentatively explained as follows. When an observer is familiar with the mask pattern, it is possible for him to detect the test pattern by looking for deviations from the expected mask pattern. In a noise-free linear system with constant increment detection. such a strategy would yield no masking at all. Since masking is observed, we postulate a compressive non-linearity which limits sensitivity, making it proportional to the 0.65 power of the mask contrast. When the observer is unfamiliar with the mask, however, he clearly cannot use this strategy. Under these conditions he must look for the actual appearance of the test itself. rather than merely finding a deviation from the expected mask pattern. This strategy is most easily understood for a noise mask. Here the observer must compare the stimulus energy at the test frequency to stimulus energies at other frequencies, rather in the manner of a statistical test. If the energy at the test frequency is sufficiently great, so that it is unlikely to have arisen in the noise pattern purely from chance. then the test is considered to be present. Thus detection occurs if the signal energy exceeds the noise by a certain ratio: if the noise contrast is increased. the signal contrast must be increased proportionally to maintain the threshold signal/noise ratio. This constitutes Weber"s Law.

Even in the case of harmonically pure mask stimuli it may be that the mask-when initially seen-is treated functionally as noise; that is. its appearance is considered to be somewhat unpredictable. With practice, of course, the observer also learns to discount harmonically pure stimuli, perhaps even more rapidly than he does broad-band stimuli. Our analysis of detection in the presence of an unfamiliar mask suggests that this process is rather similar to stimulus identification. In fact, Lasley and Cohn (1981) present a very similar analysis of pattern recognition behavior in the presence of noise. and present a theorem showing that when detection is limited by noise which is distal to the system nonlinearity, then the nonlinearity becomes transparent and Weber's Law behavior is found instead. This would account for the tendency to find Weber's Law with method-of-adjustment. Without the pressure to search for the most sensitive detection criterion that forced-choice provides, the observer may well continue to treat the mask grating as "noise".

Another interesting observation from these experiments is the universality of the 0.65 power law. The fact that so very many different masking situations all yield the same power law suggests that this is a basic and relatively inescapable property of the visual system. If it were the case that the well-practiced observer is able to totally discount the mask. then we would expect no masking at all to occur. Since this is not so, it leads us to the same conclusion as Legge (1981) who decided that there is an inherent, compressive nonlinearity in the peripheral visual system which attenuates the test signal when the mask is present.

\section{CONCLUSIONS}

We find that a simple model of masking, wherein the mask stimulus simply adds functional noise to the test channel, is entirely inadequate. In particular. the criterion by which thresholds are set varies qualitat- 
ively with practice, with the type of mask, and with the psychophysical method. Simple criteria, such as threshold signal-to-noise ratio or threshold excitation of a single channel can only be used in special cases. Two threshold criteria have been elucidated. With highly familiar masks, the subject is apparently able to discount the presence of the mask by looking for changes in its appearance. Such thresholds vary as the 0.65 power of mask contrast, which we attribute to an inherent non-linearity. With unfamiliar masks, thresholds are higher and obey Weber's Law. We believe these thresholds are set by a recognition-like process which is limited by external noise.

Acknowledgements-This work was supported by $\mathrm{NIH}$ grant EY01475 and AFOSR grant 80-0045. We wish to thank Gordon Legge, who supplied us with generous criticism, as well as his unpublished data and manuscript, and Norma Graham, our referee and editor. Both individuals made substantial contributions to this effort.

\section{REFERENCES}

Blakemore C. B. and Campbell F. W. (1969) On the existence of neurones in the human visual system selectively sensitive to the orientation and size of retinal images. $\boldsymbol{J}$. Physiol. 203, 237-260.

Bodis-Wollner I., Hendley C. D. and Kulikowski J. J. (1972) Electrophysiological and pyschophysical responses to modulation of contrast of a grating pattern. Perception 1, 341-349.

Bodis-Wollner I., Hendley C. D. and Tajel M. (1973) Contrast modulation thresholds as a function of spatial frequency. J. opt. Soc. Am. 63, 1297.

Campbell F. W. and Kulikowski J. J. (1966) Orientation selectivity of the human visual system. $J$. Physiol. 187, $437-455$.

Carlson C. R. and Pica A. (1979) Invariance in sine wave contrast discrimination. Intest. Ophthal. tisual Sci. Suppl. $18,59$.

Graham N. (1977) Visual detection of aperiodic stimuli by probability summation among narrow-band channels. Vision Res. 17, 637-652.

Fiorentini A. and Berardi N. (1971) Learning in grating wave form discrimination: specificity for orientation and spatial frequency. Vision Res. 21, 1149-1158.

Kelly D. H. (1961) Visual responses to time-dependent stimuli. 1. Amplitude sensitivity measurements. J. opt. Soc. Am. 51, 422-429.

Kulikowski J.J. and Gorea A. (1978) Complete adaptation to patterned stimuli: a necessary and sufficient condition for Weber's Law for contrast. Vixinn Res. 18, 1223-1227.

Lasley D. J. and Cohn T. E. (1981) Why luminance discrimination may be better than detection. Vision Res. 21, 273-278.
Legge G. E. (1978) Sustained and transient mechanisms in human vision: temporal and spatial properties. Vision Res. 18, 69-81.

Legge G. E. (1979) Spatial frequency masking in human vision: binocular interactions. $J$. opt. Soc. Am. 69, $838-847$.

Legge G. E. (1981) A power law for contrast discrimination. Vision Res. 21, 457-467.

Legge G. E. and Foley J. M. (1980) Contrast masking in human vision. J. apt. Soc. Am. 70, 1458-1471.

Legge G. E., Cohen W. A. and Stromeyer C. F. (1978) Spatial-frequency masking with briefly pulsed patterns. Perception 7, 161-166.

Mostafavi H. and Sakrison D. J. (1976) Structure and properties of a single channel in the human visual system. Vision Res. 16, 957 968.

Nachmias J. and Rogowitz B. E. (1982) Masking by spatially modulated gratings. Invest. Ophthal. risual Sci. Suppl. 22, 207.

Nachmias J. and Sansbury R. V. (1974) Grating contrast: discrimination may be better than detection. Vision Res. 14, 1039-1042.

Nachmias J. and Weber A. (1975) Discrimination of simple and complex gratings. Vision Res. 15, 217-223.

Pantle A. (1974) Visual Information Processing of Complex Imagery. Aerospace Medical Laboratory. Wright-Patterson AFB, Ohio No. AMRL-TR-74-43.

Pollen H. and Roehrig H. (1970) Effect of noise on the modulation transfer function of the visual channel. Vision Res. 60, 842-848.

Quick R. F. (1974) A vector-magnitude model of contrast detection. Kybernetik 16, 65-67.

Rogowitz B. E. (1977) Backward masking with sinusoidal gratings: a look at spatial frequency and temporal response. Presented to ARVO, Sarasota. Florida. May, 1977.

Sachs M. B., Nachmias J. and Robson J. G. (1971) Spatial frequency channels in human vision. $J$. opt. Soc. Am. 61, 1176-1186.

Sansbury R. V. (1974) Some properties of spatial channels revealed by pulsed simultaneous masking. Doctoral Dis. sertation, University of Pennsylvania.

Smith R. A. and Swift D. J. (1978) Simultancous masking and the bandpass of spatial-frequency channels. Presented to ARVO. Sarasota. Florida, May, 1978.

Stromeyer C. and Julesz. B. (1972) Critical bands in spatial frequency masking. J. opt. Soc. Am. 62, 1221-1230.

Swift D., Smith R. A. and Panish S. (1979a) Spatial frequency adaptation and psychophysical method. Presented to the American Psychological Association. September, 1979.

Swift D. J., Smith R. A and Panish S. (1979b) A new technique of subthreshold additivity. $J$. opt. Soc. Am. 69 , 1444.

Tolhurst D. J, and Barfield L. P. (1978) Interactions between spatial frequency channels. Vision Res. 18, $951-958$.

Wilson H. R. and Bergen J. R. (1979) A four mechanism model for threshold spatial vision. Vision Res. 19, 19-32. 